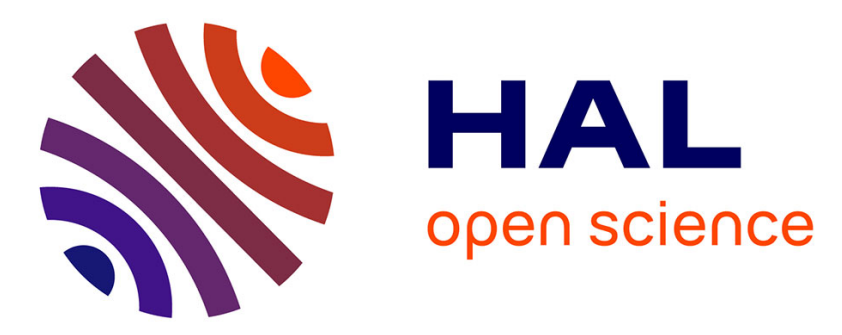

\title{
Alimentation azotée et croissance de la fétuque élevée. I. - Assimilation du carbone et répartition entre organes
} François Gastal, Bernard Saugier

\section{To cite this version:}

François Gastal, Bernard Saugier. Alimentation azotée et croissance de la fétuque élevée. I. - Assimilation du carbone et répartition entre organes. Agronomie, 1986, 6 (2), pp.157-166. hal-00884860

\section{HAL Id: hal-00884860 https://hal.science/hal-00884860}

Submitted on 1 Jan 1986

HAL is a multi-disciplinary open access archive for the deposit and dissemination of scientific research documents, whether they are published or not. The documents may come from teaching and research institutions in France or abroad, or from public or private research centers.
L'archive ouverte pluridisciplinaire HAL, est destinée au dépôt et à la diffusion de documents scientifiques de niveau recherche, publiés ou non, émanant des établissements d'enseignement et de recherche français ou étrangers, des laboratoires publics ou privés. 


\section{Alimentation azotée et croissance de la fétuque élevée. I. - Assimilation du carbone et réparti- tion entre organes}

François GASTAL (1) \& Bernard SAUGIER

Laboratoire d'Ecologie végétale, Bâtiment 362, Université de Paris-Sud, F 91405 Orsay

RÉSUMÉ L'effet de la disponibilité en azote du milieu sur la croissance de la fétuque élevée a été étudié en relation avec
le niveau de nutrition carbonée pendant une phase de repousse après une coupe.
Les cultures ont été réalisées en conditions contrôlées, à deux concentrations en azote de la solution nutritive,
combinées à deux niveaux d'éclairement.
Pour les deux éclairements de culture, la carence en azote réduit la vitesse de croissance des organes aériens.
Pendant la première moitié de la repousse, la carence en azote limite l'assimilation de carbone du couvert en
réduisant la croissance de la surface foliaire, sans modifier les capacités photosynthétiques des feuilles. Dans la
seconde moitié de la repousse, cet effet s'estompe et il apparaît une légère diminution des capacités photo-
synthétiques lorsque la teneur en N Kjeldahl des limbes tombe en dessous de $2,2 \mathrm{p}$. 100 ; cette diminution est
en partie compensée par une réduction des coûts respiratoires des organes aériens.
D'autre part, la carence en azote se traduit, par rapport au témoin bien alimenté en azote, par une augmenta-
tion de la biomasse racinaire à éclairement fort, mais par une diminution, à éclairement faible. Ce comporte-
ment illustre l'importance des interactions entre nutritions azotée et carbonée dans la croissance de la plante.

Mots clés additionnels : Conditions contrôlées, éclairement, repousse.

Nitrogen nutrition and growth of tall fescue. I. - Carbon assimilation and partitioning between organs.

The effect of nitrogen availability in nutrient solution on growth of tall fescue has been studied in relation to the level of carbon assimilation during regrowth. Plants were grown in controlled conditions at two nitrogen concentrations combined with two light levels. At the two light levels, nitrogen limitation decreased shoot growth rate. During the first half of regrowth, nitrogen deficiency limited carbon assimilation of the canopy by reducing growth in leaf area with no change in leaf photosynthetic capacity. In the second half of regrowth, the difference in growth of leaf area was reduced but a slight decrease in leaf photosynthesis appeared when the nitrogen (Kjeldahl) content of the lamina fell below $2.2 \%$ of dry matter. Such a decrease was partly compensated by a reduction in shoot respiratory cost. Nitrogen deficiency also induced an increase in root biomass with respect to the control at high light level. The reverse was observed at low light level. This behaviour stresses the importance of the interactions between nitrogen and carbon nutrition in plant growth.

Additional key words : Controlled conditions, light level, regrowth.

\section{INTRODUCTION}

Dans le cas des graminées fourragères, la production agronomique est directement liée à la vitesse de croissance des organes aériens. Celle-ci résulte de l'action combinée des facteurs physiques du milieu et des conditions nutritionnelles.

(1) Adresse actuelle : I.N.R.A., Station d'Agronomie d'Angers, Antenne expérimentale au Centre de recherches de Lusignan, F 86600 Lusignan.
Le travail présenté ici s'intéresse au second point et a pour objectif de préciser, en conditions contrôlées, les relations entre nutrition azotée, assimilation du carbone et croissance.

Les nombreux travaux antérieurs étudiant l'effet du niveau de nutrition sur le fonctionnement de la plante ont souvent privilégié l'un de ces aspects : incidence de l'alimentation en azote sur son métabolisme par le végétal et la croissance en matière sèche sans étude simultanée du niveau de nutrition carbonée, ou bien étude de l'assimilation et de la répartition du carbone 
sans considérer cette fois le métabolisme azoté. Cependant les données récentes de la physiologie mettent en lumière l'importance des interactions métaboliques entre ces 2 éléments (BASSHAM et al., 1981). De plus, on peut penser que, lors d'une repousse après une coupe, l'incidence de ces interactions sur la croissance doit être amplifiée par la dépendance plus ou moins prolongée de la croissance vis-à-vis des réserves carbonées des chaumes et des racines.

Ces considérations nous ont amenés à étudier la croissance de la fétuque élevée à 2 niveaux de nutrition azotée combinés à 2 intensités d'éclairement. Cette $1^{\text {re }}$ partie est consacrée à la description de la croissance en matière sèche, à sa répartition entre organes et à l'étude de l'assimilation du carbone.

Il a souvent été montré qu'une disponibilité en $\mathrm{N}$ limitée privilégie la croissance des racines par rapport à celle des organes aériens (BLANC, 1959 ; OSWALT et al., 1959 ; BROUWER, 1962 ; LEMAIRE, 1975 ; CALOIN et al., 1980) tandis qu'un niveau d'éclairement trop faible agit en sens opposé (TROUGHTON, 1956 ; BROUWER, 1962 ; EVANS, 1971 ; HUNT, 1975). La carence en azote peut limiter l'assimilation du carbone en réduisant la vitesse de croissance de l'appareil foliaire mais peut également diminuer, pour une surface foliaire donnée, les capacités de fixation du $\mathrm{CO}_{2}$ (YosHIDA \& CORONEL, 1976 ; ROBSON \& PARSONS, 1978 ; DE JONG, 1982 ; EVANS, 1983).

Nous essaierons dans cette étude de montrer l'importance relative de ces effets d'origine différente dans l'explication globale de la vitesse de croissance pour le cas particulier d'une repousse après une coupe.

\section{MATÉRIEL ET MÉTHODES}

\section{A. Conditions de culture et d'expérimentation}

Les cultures ont été menées en 3 essais successifs suivant un protocole identique depuis la germination jusqu'à la $2^{e}$ coupe. A ce moment, qui sera par la suite considéré comme l'instant initial de l'expérimentation, les plantes de chaque essai sont séparées en 3 lots, chacun étant soumis alors à un régime particulier d'éclairement et de nutrition azotée. Le tableau 1 résume les conditions de croissance imposées durant les cultures préliminaires et l'expérimentation proprement dite.

En serre (début du $1^{\text {er }}$ essai), la température de l'air varie de 15 à $20^{\circ} \mathrm{C}$, son humidité est incontrôlée et l'éclairage naturel est complété à $14 \mathrm{~h}$ par jour. En chambre climatisée, la température de l'air est de $22^{\circ} \mathrm{C}$ et l'humidité relative de 70 p. 100 . La photopériode est fixée à $14 \mathrm{~h}$ pour éviter la mise à fleurs.

Pour chaque essai, 3 bacs de 42 plantes, d'une surface de $0,42 \mathrm{~m}^{2}$ chacun, ont été installés dans un caisson d'éclairage, équipé de lampes OSRAM (HQI $400 \mathrm{~W}$ ), entouré de parois réfléchissantes et correctement ventilé. Ce dispositif est lui-même placé dans la chambre climatisée. Il permet d'obtenir un éclairement de croissance relativement élevé (niveau $\mathrm{E}$ dans la suite du texte), indispensable pour que l'effet de la disponibilité en azote puisse s'exprimer sur la croissance, et d'interposer des écrans pour obtenir un niveau inférieur (niveau e). Les valeurs d'éclairement mesurées en un point particulier de la surface de culture ne s'écartent pas de \pm 10 p. 100 des valeurs moyennes données dans le tableau 2 .

La composition minérale des 2 solutions nutritives utilisées, $\mathrm{N}(8 \mathrm{mM}$ de $\mathrm{N})$ et $\mathrm{n}(0,5 \mathrm{mM}$ de $\mathrm{N})$ est également indiquée dans ce même tableau.

\section{B. Techniques de mesure}

\section{Mesures de croissance}

Sur chaque bac de culture, des prélèvements de 5 plantes ont été réalisés le jour de la $2^{\mathrm{e}}$ coupe et, ensuite, avec une périodicité de 7 à $11 \mathrm{j}$ pendant 6 semaines de repousse. Un prélèvement représente 1 ligne de "culture " par bac, si bien que toutes les autres plantes restent en place et à une densité équivalente à celle du début de l'expérimentation. Le tirage

TABLEAU 1

Conditions de croissance.

Growth conditions.

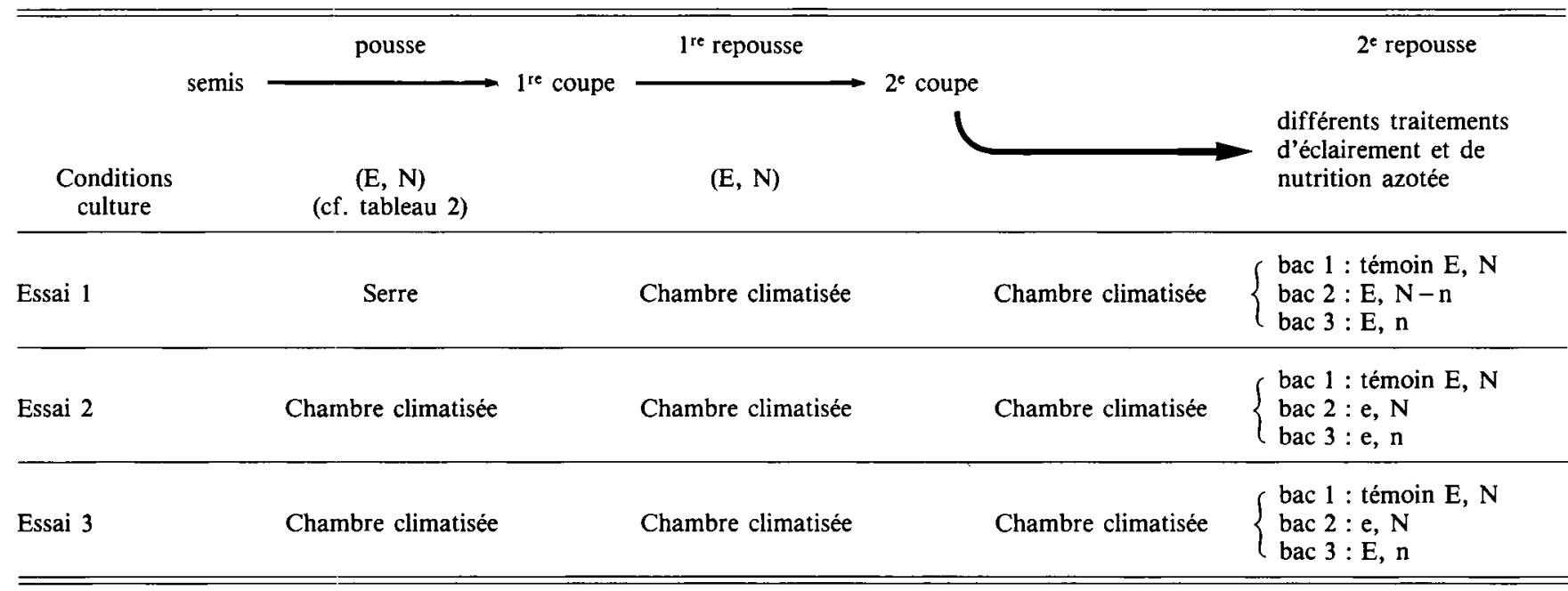


TABLEAU 2

Valeur des éclairements ( $\mu$ mol. $\mathrm{m}^{-2} \cdot \mathrm{s}^{-1}$ ) et composition minérale des solutions nutritives (meq) pendant les cultures et l'expérimentation.

Light levels (photon fluence rate, $\mu \mathrm{mol} . \mathrm{m}^{-2} . \mathrm{s}^{-1}$ ) and mineral composition of nutrient medium (meq) during growth.

Eclairement de croissance :

\begin{tabular}{lcc}
\cline { 2 - 3 } & $\begin{array}{c}30 \mathrm{~cm} \text { au-dessus } \\
\text { du collet }\end{array}$ & $\begin{array}{c}\text { à hauteur } \\
\text { du collet }\end{array}$ \\
\hline éclairement $\mathrm{E}$ & 450 & 310 \\
\hline éclairement $\mathrm{e}$ & 250 & 180 \\
\hline
\end{tabular}

Solutions nutritives :

\begin{tabular}{lcc}
\cline { 2 - 3 } & ${ }^{*} \mathrm{~N}: 8 \mathrm{mM}(\mathrm{N})$ & $* * \mathrm{n}: 0,5 \mathrm{mM}(\mathrm{N})$ \\
\hline $\mathrm{KNO}_{3}$ & 1,9 & 0,05 \\
$\mathrm{Ca}\left(\mathrm{NO}_{3}\right)_{2}$ & 1,1 & 0,15 \\
$\mathrm{NO}_{3} \mathrm{NH}_{4}$ & 2,5 & 0,15 \\
$\mathrm{CaCl}$ & 0,5 & 1,45 \\
$\mathrm{NaCl}_{\mathrm{MgSO}}$ & 0,1 & - \\
$\mathrm{MgSO}_{4}$ & 0,5 & 0,5 \\
$\mathrm{~K}_{2} \mathrm{SO}_{4}$ & - & 0,7 \\
$\mathrm{Na}_{2} \mathrm{SO}_{4}$ & - & 0,1 \\
$\mathrm{PO}_{4} \mathrm{H}_{2} \mathrm{~K}$ & 0,4 & 1,05 \\
$\mathrm{PO}_{4} \mathrm{HK}_{2}$ & 0,3 & 0,8 \\
\hline \hline
\end{tabular}

+ oligo-éléments

* N : solution nutritive pendant 14 jours après la coupe. ** $\mathrm{n}$ : solution nutritive pour la suite de la repousse.
« au hasard » est ici exclu sous peine de bouleverser entièrement la structure du couvert. Du fait de la mortalité de certains individus après la coupe, la surface disponible par plante durant l'expérimentation est supérieure à celle de la période d'implantation et évaluée à $1,3 \mathrm{dm}^{2}$.

Pour chaque individu prélevé, nous avons séparé racines, "chaumes " (gaines comprises entre le plateau de tallage et la hauteur de coupe, soit $5 \mathrm{~cm}$ ) et " parties récoltables ", comprenant elles-mêmes limbes verts, gaines au-dessus de la hauteur de coupe et éventuellement débris de limbes.

Sur chacune de ces fractions de plante, ont été déterminés le poids frais et le poids de matière séchée à $65^{\circ} \mathrm{C}$. Les surfaces des limbes verts ont été mesurées avec un planimètre automatique Delta $T$ Devices.

\section{Mesures d'échanges gazeux}

Le système de mesure a été conçu pour évaluer en continu et sur $24 \mathrm{~h}$ les échanges de $\mathrm{CO}_{2}$ des parties aériennes et racinaires séparément. Il comprend (cf. fig. 1) :

- un compartiment qui renferme les parties aériennes de la plante, C.A., clos et régulé en température, humidité relative et teneur en $\mathrm{CO}_{2}$;

- un compartiment qui contient le système racinaire de la plante, C.R., séparé de C.A. par un joint de mastic Terosta, et comprenant la solution nutritive et une phase gazeuse. Ce compartiment est également clos, mais le bocal racinaire est prolongé par un volume tampon d'air, extérieur à la cellule. Une circu-

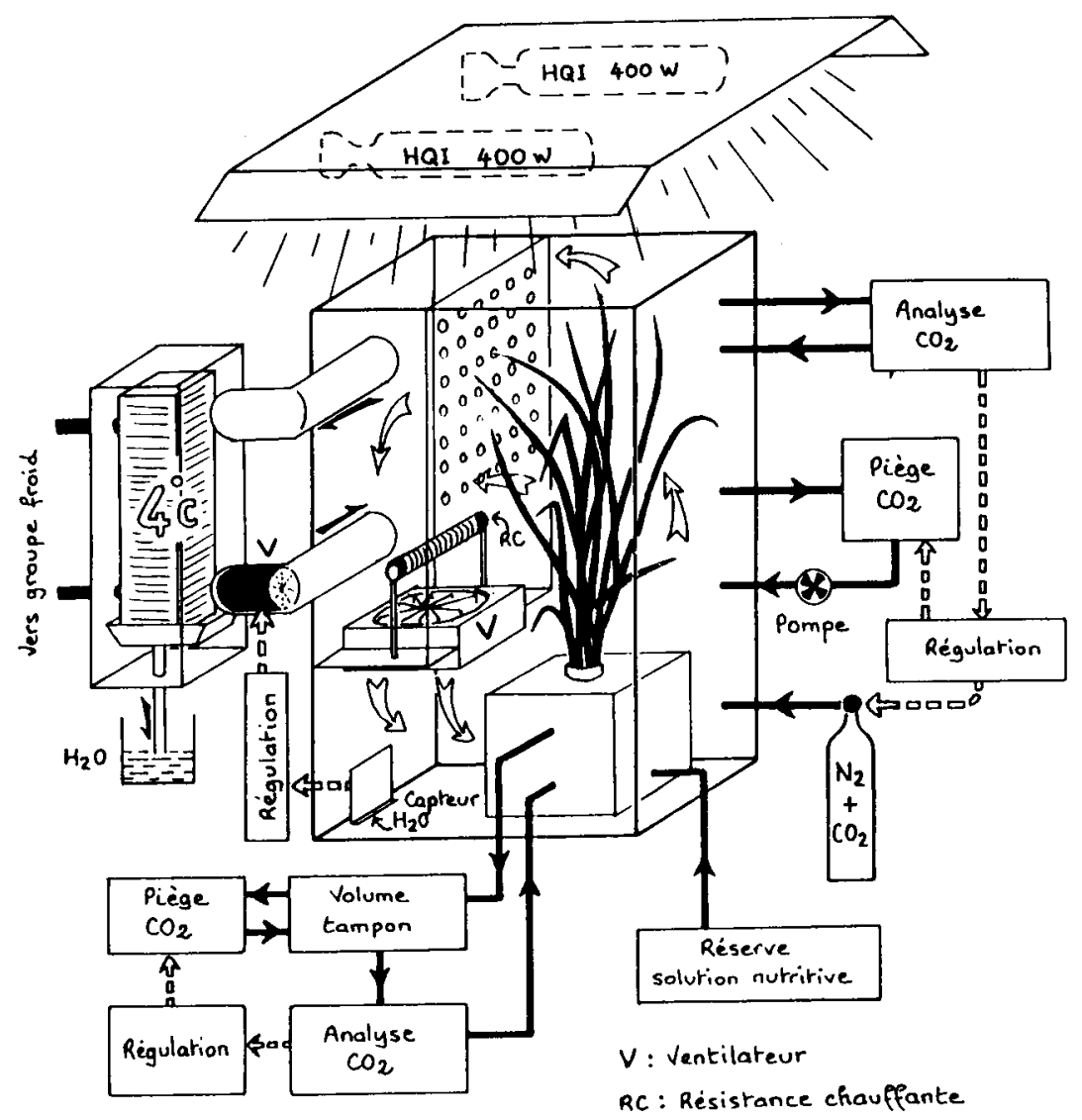


lation d'air entre ce volume tampon et une rampe de bullage dans le bas du bocal racinaire permet l'aération de la solution nutritive.

Les concentrations en $\mathrm{CO}_{2}$ sont mesurées avec un analyseur de $\mathrm{CO}_{2}$ ADC MKIII, qui reçoit alternativement une fraction de l'air de C.A. et de C.R. La régulation s'effectue automatiquement par piégeage du $\mathrm{CO}_{2}$ de l'air sur une colonne de chaux sodée lorsque la teneur en $\mathrm{CO}_{2}$ excède $340 \mathrm{ppm}$, ou bien, pour C.A., par injection d'un volume connu de $\mathrm{CO}_{2}$ gazeux lorsque la teneur en $\mathrm{CO}_{2}$ est inférieure à $320 \mathrm{ppm}$. Le nombre d'injections ou le temps de piégeage nécessaire pour maintenir la concentration en $\mathrm{CO}_{2}$ de l'air de chacun des 2 compartiments à une valeur déterminée $( \pm 20 \mathrm{ppm})$ permettent la mesure de l'assimilation ou du rejet de carbone par la plante. La quantité de $\mathrm{CO}_{2}$ dissous dans la solution nutritive est considérée comme négligeable par rapport à celle dégagée par le système racinaire sur un intervalle de temps supérieur à l'heure au $\mathrm{pH}$ où nous avons travaillé (de l'ordre de 6). La précision absolue des mesures peut être estimée à 5-10 p. 100 dans le compartiment aérien et à $10-15$ p. 100 dans le compartiment racinaire.

L'humidité relative de C.A. est maintenue constante à 70 p. 100 ( \pm 7 à 8 p. 100) par passage de l'air sur un condenseur maintenu à $4{ }^{\circ} \mathrm{C}$. La mesure est faite par un capteur capacitif Vaisala. L'eau de condensation récupérée permet de déterminer la transpiration sur quelques heures.

Le volume de solution nutritive dans le bocal racinaire est maintenu constant par compensation automatique à l'aide d'une pompe péristaltique.

Lors de l'essai 1, l'éclairement dans cette enceinte (1 $100 \mu \mathrm{mol} . \mathrm{m}^{-2} \cdot \mathrm{s}^{-1}$ à $30 \mathrm{~cm}$ au-dessus du collet, $450 \mu \mathrm{mol} . \mathrm{m}^{-2} . \mathrm{s}^{-1}$ à hauteur du collet) était supérieur à l'éclairement de croissance. De plus, seuls les échanges de $\mathrm{CO}_{2}$ des parties aériennes ont pu être mesurés. En revanche, lors des essais 2 et 3, l'intensité lumineuse de la cellule de mesure à $30 \mathrm{~cm}$ au-dessus du collet a été ramenée à une valeur très proche de celle des bâtis de culture ( 450 ou $250 \mu \mathrm{mol} . \mathrm{m}^{-2}$. $\mathrm{s}^{-1}$ ), et les échanges de $\mathrm{CO}_{2}$ des racines et des parties aériennes ont pu être mesurés.

$\mathrm{Au}$ cours de la période d'expérimentation ( $2^{\mathrm{e}}$ repousse), une plante a été régulièrement prélevée, dans l'un des 3 bacs du système de culture et installée dans l'enceinte de mesure. Après une journée considérée comme un laps de temps suffisant pour l'adaptation de la plante à de nouvelles conditions de crois- sance, les mesures d'échanges de $\mathrm{CO}_{2}$ ont été enregistrées pendant $24 \mathrm{~h}$.

Les enregistrements, intégrés sur un cycle nycthéméral, ont permis d'établir le bilan carboné sur $24 \mathrm{~h}$ de la plante étudiée. Toutefois, cette dernière bénéficie dans la cellule de mesure d'une surface au sol plus importante $\left(2,5 \mathrm{dm}^{2}\right)$ qu'en culture, les valeurs obtenues ne peuvent pas être reliées directement aux vitesses de croissance déterminées par l'accroissement de matière sèche.

\section{RÉSULTATS ET INTERPRÉTATION}

\section{A. Croissance après la coupe}

L'évolution de la biomasse au cours de la croissance est présentée dans la figure 2. A éclairement fort et nutrition azotée non limitante $(E, N)$, la matière sèche aérienne récoltée après 6 semaines de repousse est d'environ $10 \mathrm{~g} /$ plante (fig. $2 \mathrm{~A}$ et $2 \mathrm{~B}$ ) et l'indice foliaire (non représenté) atteint des valeurs élevées, de l'ordre de 16. Ce dernier résultat est inhabituel pour l'agronome (au champ, les valeurs les plus élevées sont de l'ordre de 8 à 10). Il est cependant retrouvé en culture dense sur milieu liquide par ROBSON \& PARSONS (1978), PARSONS \& ROBSON (1981). A titre indicatif, l'extrapolation de la biomasse aérienne à une culture aboutirait à une production de 7 à $8 \mathrm{t} /$ ha. Dans ce cas, la précision des valeurs obtenues est évidemment limitée par la petite taille du couvert étudié et les effets de bordure.

La reprise de croissance des parties aériennes après la coupe est plus rapide dans l'essai 1 que dans l'essai 2. Toutefois, à partir d'une dizaine de jours après la coupe, les pentes des courbes de croissance des témoins $(\mathrm{E}, \mathrm{N})$ des 2 essais sont tout à fait similaires. Cette différence de vitesse de redémarrage peut être attribuée à un niveau de réserves carbonées plus important (DAVIES, 1965) pour les plantes de l'essai 1, dont la croissance antérieure à la $2^{\mathrm{e}}$ coupe a probablement été moins rapide (température en serre plus basse que dans la salle de culture) (BOOYSEN \& NELSON, 1975). Toutefois, la biomasse des chaumes après coupe est comparable dans les 2 essais (tabl. 3 ).

Comme attendu, la limitation de l'éclairement ou de la quantité d'azote disponible dans le milieu nutritif conduit à une diminution de la vitesse de croissance des parties aériennes (fig. $2 \mathrm{~A}$ et $2 \mathrm{~B}$ ). Toutefois,

TABLEAU 3

Variations de la biomasse des chaumes dans les divers traitements.

Variations in stubble biomass in the various treatments.

\begin{tabular}{|c|c|c|c|c|c|c|c|}
\hline Jours après coupe & $\mathrm{E}, \mathrm{N}$ & $\mathrm{E}, \mathrm{N}-\mathrm{n}$ & $\mathrm{E}, \mathrm{n}$ & Jours après coupe & $\mathrm{E}, \mathrm{N}$ & $\mathrm{e}, \mathrm{N}$ & $\mathrm{e}, \mathrm{n}$ \\
\hline 0 & 1,15 & - & - & 0 & 1,29 & - & - \\
\hline 7 & 1,41 & - & 1,20 & 12 & 1,70 & 1,29 & 1,16 \\
\hline 14 & 1,92 & - & 1,10 & 21 & 1,75 & 1,44 & 0,93 \\
\hline 21 & 2,06 & 1,54 & 1,15 & 30 & 1,81 & 1,44 & 1,00 \\
\hline 28 & 1,85 & 1,92 & 1,52 & 41 & 2,20 & 1,61 & 1,13 \\
\hline 35 & - & 2,15 & 1,57 & & & & \\
\hline 42 & 2,20 & 2,40 & 2,30 & & & & \\
\hline
\end{tabular}




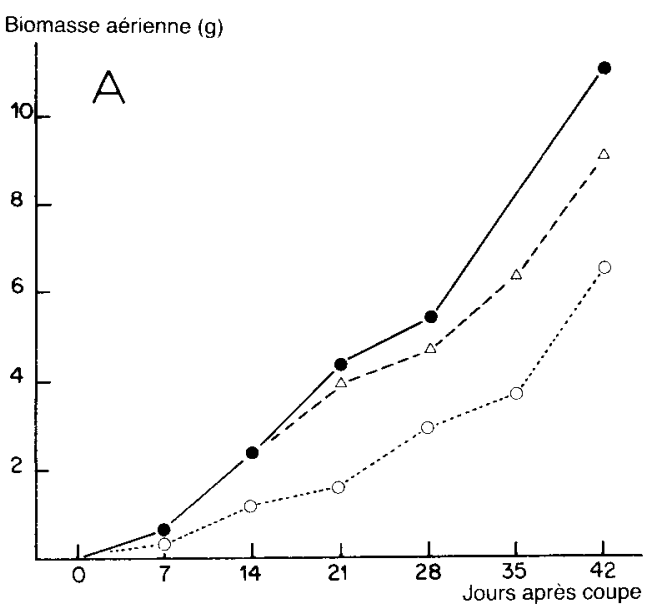

Biomasse aérienne $(\mathrm{g})$

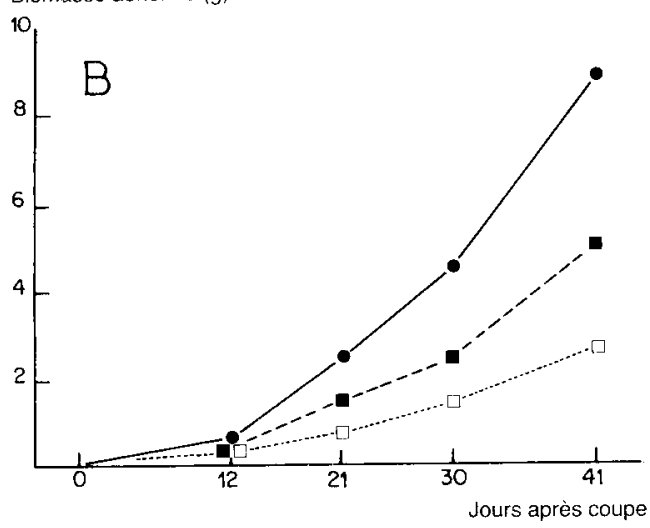

Figure 2

Evolution de la biomasse au cours de la croissance.

$A:$ évolution de la biomasse aérienne (essai l).

$B$ : évolution de la biomasse aérienne (essai 2).

$C$ : évolution de la biomasse racinaire (essai l).

$D$ : évolution de la biomasse racinaire (essai 2).

- : $(E, N) ; \quad:(E, N-n) ; O:(E, n) ; \square:(e, n) ; \mathbf{0}:(e, N)$. Eclairement incident pendant la croissance : $E\left(450 \mathrm{\mu Mm}^{-2} \mathrm{~s}^{-1}\right)$ ou e $\left(250 \mu \mathrm{Mm}^{-2} \mathrm{~s}^{-1}\right)$.

Concentration en azote de la solution nutritive: $N(8 \mathrm{mM})$ ou $n(0,5 \mathrm{mM})$

à éclairement élevé et nutrition azotée limitante $(E, n)$, cette diminution est plus marquée en début qu'en fin de repousse.

La carence en azote entraîne une augmentation spectaculaire de la biomasse racinaire à éclairement élevé (fig. 2C), non seulement en valeur relative par rapport à la biomasse totale comme cela a été fréquemment signalé (voir introduction), mais également en valeur absolue. Cette stimulation de la croissance racinaire intervient plus rapidement si la mise en carence a lieu à la $3^{\mathrm{e}}$ semaine de repousse $(\mathrm{E}, \mathrm{N}-\mathrm{n})$ que juste après la coupe $(E, n)$.

A éclairement plus faible, la comparaison de $(e, N)$ et $(e, n)$ montre que la carence en azote se traduit alors par une diminution de la biomasse racinaire (fig. 2D). Dans le cas d'une repousse après coupe, l'effet de la disponibilité en azote du milieu nutritif sur la balance biomasse aérienne/biomasse racinaire est donc totalement dépendant de la disponibilité en carbone.

La biomasse des chaumes (tabl. 3) varie de façon analogue à celle des racines dans les traitements bien alimentés en azote; elle augmente tardivement dans le
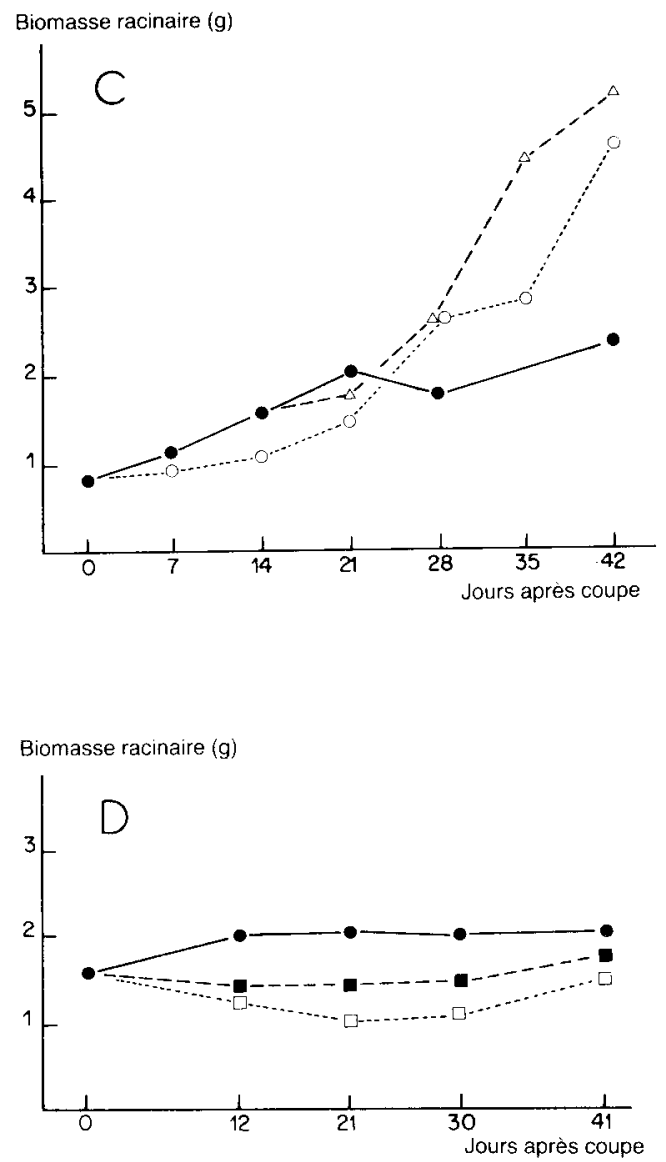

Biomass development during growth.

$A$ : shoot biomass (experiment 1 ).

$B$ : shoot biomass (experiment 2)

$C$ : root biomass (experiment 1 ).

$D$ : root biomass (experiment 2 ).

Incident light level during growth : E (450 $\left.\mathrm{\mu Mm}^{-2} \mathrm{~s}^{-1}\right)$ or e $\left(250 \mu \mathrm{Mm}^{-2} \mathrm{~s}^{-1}\right)$.

Nitrogen concentration in the nutrient solution: $N(8 \mathrm{mM})$ or $n(0.5 \mathrm{mM})$.

traitement $(E, n)$ et reste à peu près constante dans le traitement $(\mathrm{e}, \mathrm{n})$.

La masse surfacique des limbes des graminées augmente au cours de la croissance, en conditions d'environnement stables (fig. 3). De ce fait, nous avons

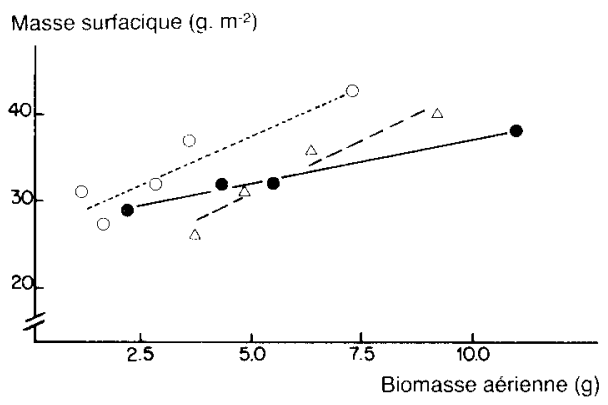

Figure 3

Evolution de la masse surfacique au cours de la croissance (essai 1 ) en fonction de la biomasse aérienne produite.

- : $(E, N) ; \Delta:(E, N-n) ; O:(E, n)$.

Specific leaf weight during growth (experiment 1) as a function of shoot biomass production. 
comparé son évolution entre les traitements, non pas à temps de repousse égal mais en fonction de l'état de croissance (biomasse) de l'appareil aérien. Il apparaît ainsi une augmentation du poids sec par unité de surface des limbes dans le cas d'une carence en azote. Deux hypothèses peuvent être avancées : d'une part, il est connu qu'une déficience en azote entraîne une accumulation de sucres solubles et éventuellement d'amidon dans les limbes (DEINUM \& DIRVEN, 1975) ; d'autre part, ces mêmes auteurs indiquent, dans ces conditions, une augmentation de la teneur en cellulose et lignine des limbes, ce qui impliquerait l'épaississement des parois de certaines cellules.

\section{B. Assimilation du carbone}

De manière à pouvoir comparer les capacités photosynthétiques et pour s'affranchir des différences de surface foliaire entre les plantes des différents traitements, nous avons porté dans la figure 4 la quantité globale de $\mathrm{CO}_{2}$ assimilé pendant la période lumineuse en fonction de l'indice foliaire (mesures de l'essai 1). Cette quantité a été déterminée en divisant la surface foliaire par la surface de sol occupée par la plante dans la chambre de mesure $\left(2,5 \mathrm{dm}^{2}\right)$.

Il apparaît que, sauf pour les points entourés (fig. 4) obtenus à la fin de la période de repousse dans des plantes cultivées sur milieu pauvre en azote $(E, n)$, la carence en azote a été sans effet apparent sur les capacités de fixation du carbone, bien que les teneurs en $\mathrm{N}$ Kjeldahl aient chuté de $5 \mathrm{p} .100$ en début de repousse à $1,9 \mathrm{p} .100$ à la $6^{\mathrm{e}}$ semaine après la coupe (GASTAL \& SAUGIER, 1986).

En revanche, à biomasse aérienne équivalente, le rapport respiration des organes aériens/fixation

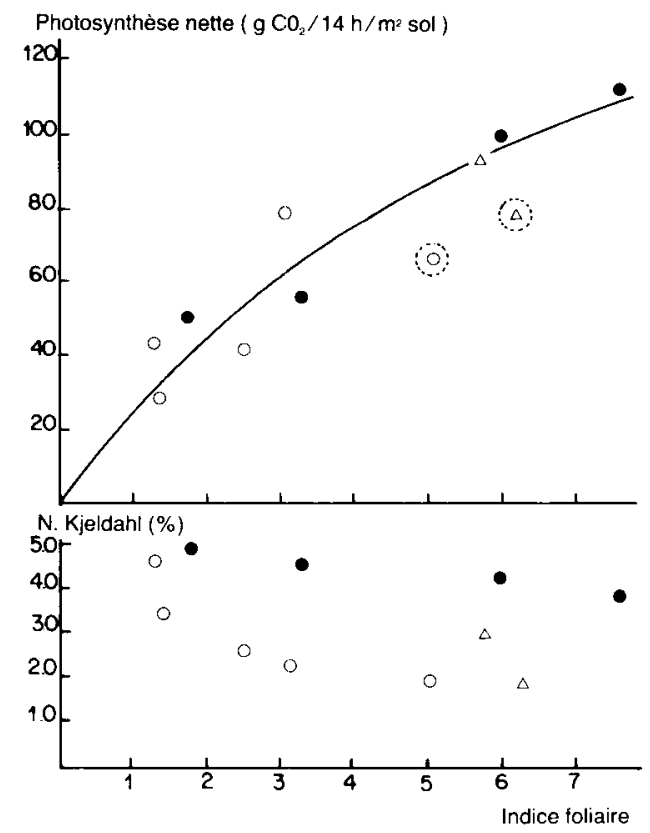

Figure 4

Assimilation diurne du $\mathrm{CO}_{2}$ par les parties aériennes (essai l) en fonction de l'indice foliaire et teneur en $\mathrm{N}$-Kjeldahl des limbes correspondants.

$\bullet:(E, N) ; \triangle:(E, N-n) ; O:(E, n)$.

Assimilation of $\mathrm{CO}_{2}$ by the shoots during the light period (experiment 1) as a function of leaf area index, and the nitrogen content (Kjeldahl) in the corresponding leaves. diurne de $\mathrm{CO}_{2}$ est nettement diminué par la carence en azote (fig. 5). Ce résultat s'accorde avec les concepts développés par MCCREE (1974) et PENNING DE VRIES (1975) selon lesquels la composante d'entretien de la respiration dépend de la teneur en protéines puisqu'une bonne part de l'énergie fournie est affectée à leur renouvellement. De plus, la carence azotée entraîne une réduction de l'assimilation de l'azote et de la dépense énergétique associée et donc de la respiration de croissance par masse de carbone assimilé.

Les mesures des échanges gazeux du traitement témoin $(\mathrm{E}, \mathrm{N})$ lors des essais 2 et 3 , faites dans des conditions d'éclairement très proches des conditions de culture, sont rapportées dans la figure 6. Elles sont

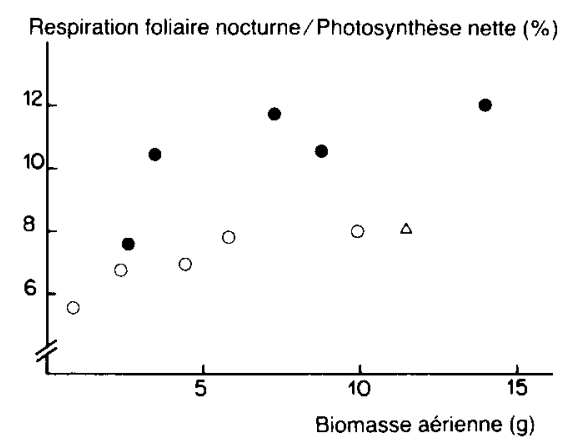

Figure 5

Evolution du rapport respiration nocturne des parties aériennes/ photosynthèse nette diurne au cours de la croissance en fonction de la biomasse aérienne produite (éclairement de mesure moyen: $\left.875 \mathrm{\mu Mm}^{-2} \mathrm{~s}^{-1}\right)$.

- :(E,N); $\triangle:(E, N-n) ; 0:(E, n)$.

Change during growth of the ratio shoot respiration during the dark period/shoot net photosynthesis during the light period as a function of the shoot biomass production (mean photon fluence rate at measurement : $875 \mathrm{\mu Mm}^{-2} \mathrm{~s}^{-1}$ ).

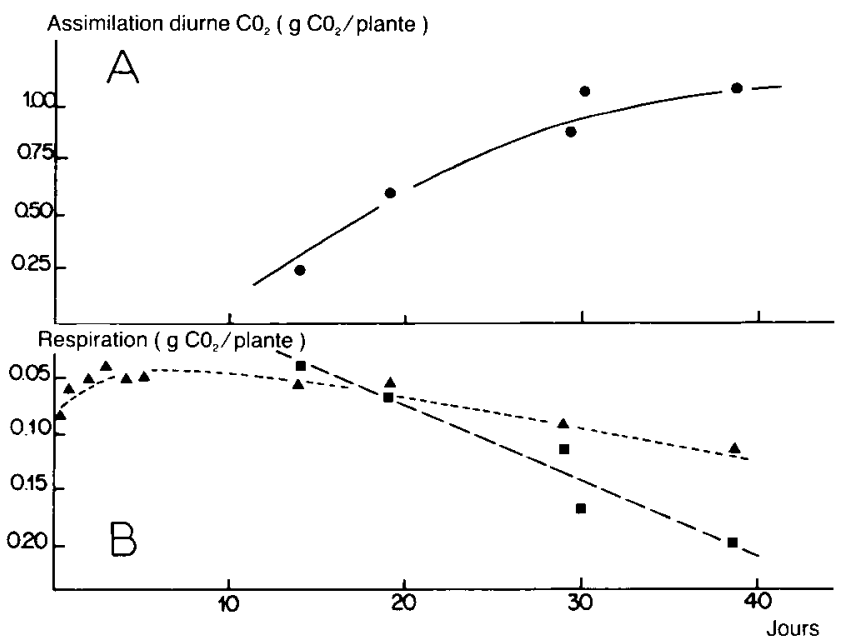

Figure 6

Echanges de $\mathrm{CO}_{2}$ du traitement témoin $(E, N)$ mesurés à l'éclairement de croissance dans les essais 2 et 3.

$A$ : assimilation diurne du $\mathrm{CO}_{2}(-$

${ }_{B}\{$ : respiration nocturne des parties aériennes $(----\square)$.

$\{$ : respiration journalière des racines (-...... $\mathbf{\Delta}$ ).

$\mathrm{CO}_{2}$ exchanges measured at the photon fluence rate for experiments 2 and 3 (treatment $E, N$ ).

$A: \mathrm{CO}_{2}$ assimilation during the light period (- $\longrightarrow$ ).

$B\{:$ shoot respiration during the dark period (- $---\square)$

$\{$ : root daily respiration ( ............ 
présentées cette fois en fonction du temps de repousse. Ainsi, les premiers jours suivant la coupe, l'assimilation du carbone est totalement arrêtée et les chaumes laissés en place montrent alors une activité respiratoire. Avec l'élongation et le développement des limbes qui étaient renfermés dans les chaumes lors de la défoliation, la fixation de $\mathrm{CO}_{2}$ reprend progressivement (fig. 6A). La suppression des organes assimilateurs - et ainsi des migrations d'assimilats carbonés vers les racines - entraîne une réduction importante de la respiration racinaire (fig. 6B), diminution déjà observée dans ces conditions par DAVIDSON \& MILTHORPE (1966) et FROSSARD (1980). Cependant, le rejet de $\mathrm{CO}_{2}$ par ces organes est maintenu à un niveau relativement élevé, 40 à $50 \mathrm{p} .100$ de sa valeur avant la coupe, ce qui entraîne un bilan carboné négatif de la plante entière les premiers jours après la coupe, résultats en accord avec ceux de CLÉMENT et al., (1978).

Les mesures effectuées sur les plantes des autres traitements seront présentées dans le paragraphe suivant.

\section{MODÉLISATION DU BILAN CARBONÉ ET COMPARAISON AVEC LES MESURES}

Les conditions expérimentales adoptées ne permettent pas de comparer directement l'assimilation carbonée telle que nous l'avons mesurée et la matière sèche élaborée lors des cultures pour 3 raisons :

- la surface au sol disponible par plante est différente entre le bâti de culture et la cellule de mesure ;

- l'extinction du rayonnement n'est pas la même dans les 2 systèmes;

- les mesures disponibles par traitement sont trop peu nombreuses.

Compte tenu de ces considérations, nous avons cherché à simuler le bilan carboné des différents traitements au cours de la repousse avec un objectif double. D'une part, cette technique permet de prouver la cohérence entre nos mesures d'assimilation du $\mathrm{CO}_{2}$ et de croissance en biomasse, d'autre part, elle permet de retracer l'évolution des échanges de $\mathrm{CO}_{2}$ des couverts des différents traitements au cours de la repousse.

Nous supposerons connue et nous utiliserons comme base de calcul l'évolution de l'indice foliaire pour chaque traitement pendant la repousse. Ceci n'est donc en rien une tentative de simulation de la croissance mais se limite aux 2 objectifs annoncés.

Pour ce faire, la démarche suivie est, dans un premier temps, le calcul sur chaque journée de croissance, d'une part, de la fixation diurne de $\mathrm{CO}_{2}$ par plante, d'autre part, des pertes respiratoires. Pour apprécier la validité des relations adoptées, les mesures d'échanges de $\mathrm{CO}_{2}$ disponibles sont comparées aux valeurs calculées compte tenu des conditions particulières de la chambre d'assimilation. Dans un second temps, la biomasse synthétisée à chaque journée de repousse est évaluée comme la différence entre l'assimilation diurne et la respiration totale de $\mathrm{CO}_{2}$. Enfin, la quantité obtenue est multipliée par $30 / 44$, ce qui suppose une teneur en carbone de la biomasse de $0,40(\mathrm{~g} / \mathrm{g})$, puis intégrée sur la période de repousse.

L'assimilation diurne de carbone du couvert est cal- culée à partir du modèle simple développé par MONTEITH (1965), ce qui nécessite la connaissance d'une part, de la réponse de la photosynthèse de la feuille à l'éclairement et, d'autre part, d'un facteur « s » représentant la fraction de rayonnement non intercepté par un couvert d'indice foliaire égal à 1 , qui dépend de son architecture. La relation photosynthèse-éclairement de la feuille est ajustée à une hyperbole non équilatère (CHARTIER \& BETHENOD, 1977) à partir de mesures au laboratoire sur la dernière feuille adulte de plusieurs talles (GASTAL, 1984). La valeur de s adoptée $(0,7)$ est une donnée bibliographique propre à un couvert de graminées (WOLEDGE \& LEAFE, 1976 ; PARSONS \& ROBSON, 1981). Toutes les valeurs des paramètres utilisés pour les calculs sont indiquées dans le tableau 4.

La quantité journalière de carbone perdu par respiration pour la plante entière $(R)$ est évaluée à partir de la relation $R=a P_{B}+b M(M C C R E E, 1974)$ où $M$ est la biomasse totale et $P_{B}$ la photosynthèse brute. Pour obtenir $P_{B}$, nous avons ajouté la respiration de la plante entière à la photosynthèse nette, en supposant que la respiration des parties aériennes a la même intensité le jour que la nuit. Un coefficient réducteur, fonction de la teneur en $\mathrm{N}$ Kjeldahl de la matière sèche, est multiplié au coefficient d'entretien $b$ pour tenir compte de la diminution de la teneur en protéine lors de la carence azotée. Ce coefficient est évalué d'après PENNING DE VRIES (1975). Les valeurs adoptées sont également indiquées dans le tableau 4.

Les valeurs calculées de l'assimilation diurne du $\mathrm{CO}_{2}$ montrent un bon accord avec les mesures en chambre d'assimilation (fig. 7 et tabl. 5). Il en est de même pour les valeurs calculées et mesurées de respiration (tabl. 5), bien que les valeurs adoptées pour les coefficients de croissance et d'entretien (tabl. 4) soient relativement peu élevées en comparaison avec celles rapportées dans la littérature (RUGET, 1981).

\section{TABLEAU 4}

Paramètres utilisés pour les calculs de photosynthèse et respiration.

Parameter values used for computing photosynthesis and respiration.

1) Photosynthèse feuille :

$$
\left[\begin{array}{rl}
\mathbf{P}_{\mathrm{B}}= & \frac{\alpha \mathrm{E}+\mathrm{P}_{\mathrm{m}}-\sqrt{\left(\alpha \mathrm{E}+\mathrm{P}_{\mathrm{m}}\right)^{2}-4 \mathrm{M} \alpha \mathrm{EP}}}{2 \mathrm{M}} \\
\mathbf{P}_{\mathrm{N}}= & \mathrm{P}_{\mathbf{B}}-\mathrm{R}_{0} \\
& \mathrm{P}_{\mathrm{m}}=0,95 \quad \mathrm{mg} \mathrm{CO}_{2} \mathrm{~m}^{-2} \mathrm{~s}^{-1} \\
& \alpha=0,0027 \mathrm{mg} \mathrm{CO}_{2} \mu \mathrm{E}^{-1} \mathrm{~m}^{2} \mathrm{~s} \\
& \mathrm{M}=0,85 \\
& \mathbf{R}_{0}=0,05 \quad \mathrm{mg} \mathrm{CO}_{2} \mathrm{~m}^{-2} \mathrm{~s}^{-1}
\end{array}\right.
$$

2) Paramètres du modèle MoNTEITH.

$\mathrm{S}=0,7$

$\tau=0,08$ (transmission de la feuille, $400-700 \mathrm{~nm}$ ).

3) Paramètres du calcul respiration:

$\mathrm{a}=0,20$

$\mathrm{b}=0,012 \mathrm{j}^{-1}$

coût de maintenance des protéines : $40 \mathrm{mg}$ glucose $/ \mathrm{g}$.jour soit $b_{\text {prot. }}=\% \mathrm{~N} \times 0,0022 \mathrm{~g}_{\mathrm{MS}} / \mathrm{g}_{\mathrm{MS}}$.jour. 
TABLEAU 5

Comparaison des valeurs calculées aux mesures de photosynthèse nette et de respiration de la plante entière. Comparison between calculated values and measurements of whole plant net photosynthesis and respiration.

\begin{tabular}{|c|c|c|c|c|c|c|c|c|c|}
\hline Traitement & & $\begin{array}{c}\text { Date } \\
\text { (jour depuis } \\
2^{\mathrm{e}} \text { coupe) }\end{array}$ & $\begin{array}{l}\text { Surf. fol. } \\
\quad\left(\mathrm{dm}^{2}\right)\end{array}$ & $\begin{array}{l}\text { MS tot. } \\
\text { (g) }\end{array}$ & $\frac{\mathrm{N}}{\left(\mathrm{g} / 100 \mathrm{~g}_{\mathrm{MS}}\right)}$ & $\begin{array}{l}\text { Pn mesuré } \\
\mathrm{gCO}_{2} / 14 \mathrm{~h}\end{array}$ & $\begin{array}{l}\text { Pn calc. } \\
\mathrm{gCO}_{2} / 14 \mathrm{~h}\end{array}$ & $\begin{array}{l}\mathrm{R}_{\mathrm{T}} \text { mesuré } \\
\mathrm{gCO}_{2} / 24 \mathrm{~h}\end{array}$ & $\begin{array}{c}\mathrm{R}_{\mathrm{T}} \text { calc. } \\
\mathrm{gCO}_{2} / 24 \mathrm{~h}\end{array}$ \\
\hline \multirow{5}{*}{$\mathrm{E}, \mathrm{N}$} & \multirow{3}{*}{ Essai 2} & 18 & 5,95 & 7,02 & 4,0 & 0,62 & 0,58 & 0,12 & 0,16 \\
\hline & & 29 & 10,94 & 8,58 & 3,5 & 0,87 & 0,97 & & \\
\hline & & 38 & 21,60 & 14,65 & 3,4 & 1,10 & 1,14 & 0,30 & 0,30 \\
\hline & \multirow{2}{*}{ Essai 3} & 14 & 3,1 & 3,49 & 4,1 & 0,26 & 0,30 & 0,09 & 0,08 \\
\hline & & 30 & 15,03 & 12,05 & 3,6 & 1,09 & 1,08 & 0,31 & 0,27 \\
\hline \multirow{2}{*}{$\mathrm{e}, \mathrm{N}$} & \multirow{2}{*}{ Essai 2} & 26 & 3,82 & 3,29 & 3,7 & 0,24 & 0,23 & 0,11 & 0,07 \\
\hline & & 36 & 10,5 & 7,40 & 3,6 & 0,56 & 0,58 & 0,19 & 0,16 \\
\hline \multirow[t]{2}{*}{$\mathrm{E}, \mathrm{n}$} & Essai 3 & 25 & 8,4 & 7,71 & 2,2 & 0,65 & 0,84 & 0,16 & 0,17 \\
\hline & \multirow{3}{*}{ Essai 2} & 24 & 3,9 & 3,75 & 3,4 & 0,34 & 0,23 & 0,11 & 0,07 \\
\hline \multirow[t]{2}{*}{$\mathrm{e}, \mathrm{n}$} & & 31 & 7,44 & 6,53 & 3,3 & 0,42 & 0,45 & 0,12 & 0,13 \\
\hline & & 43 & 5,9 & 4,68 & 3,0 & 0,34 & 0,36 & 0,10 & 0,09 \\
\hline
\end{tabular}

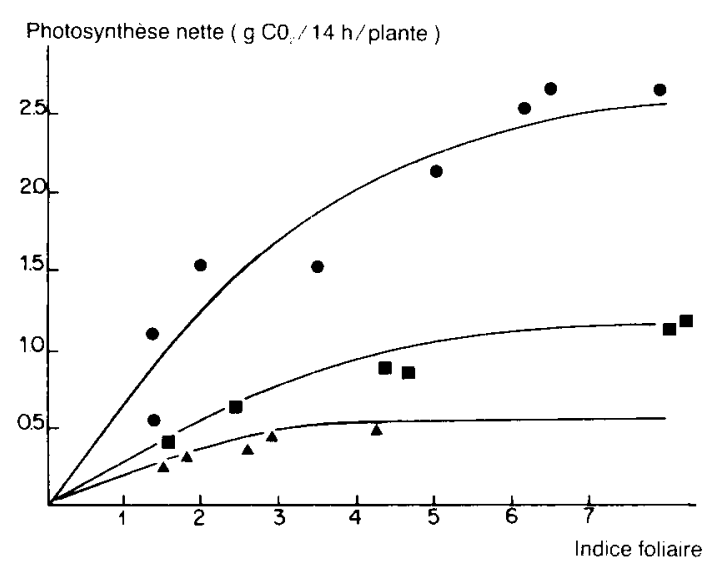

Figure 7

Comparaison des mesures de photosynthèse nette (symboles) aux valeurs calculées (courbes continues), d'après MONTEITH, 1965. - : $1175 \mu \mathrm{Mm}^{-2} \mathrm{~s}^{-1}: 450 \mu \mathrm{Mm}^{-2} \mathrm{~s}^{-1} \mathbf{\Delta}: 250 \mu \mathrm{Mm}^{-2} \mathrm{~s}^{-1}$. Comparison between the net photosynthesis measurements and the values calculated according to MONTEITH, 1965.

Notons que, du fait des perturbations du rapport biomasse aérienne/biomasse racinaire dues à la coupe et à la carence en azote, les calculs de respiration sur la plante entière sont partiellement biaisés puisque coefficients d'entretien et de croissance sont plus élevés pour les racines que pour les parties aériennes (LAMBERS, 1979). Ainsi, en début de repousse, les valeurs de respiration mesurées semblent-elles en général plus élevées que les valeurs calculées (tabl. 5).

Les biomasses calculées correspondent relativement bien aux mesures (fig. 8), compte tenu des imprécisions inévitables lors de toute mesure en absolu d'assimilation du $\mathrm{CO}_{2}$ ainsi que de certains phénomènes biologiques qui n'ont pas été pris en compte par manque de données expérimentales (exsudation racinaire en particulier).

Il est donc permis de penser que les évolutions, au cours de la repousse, des échanges de $\mathrm{CO}_{2}$ calculées pour les différents couverts et représentées figure 9 sont valides.

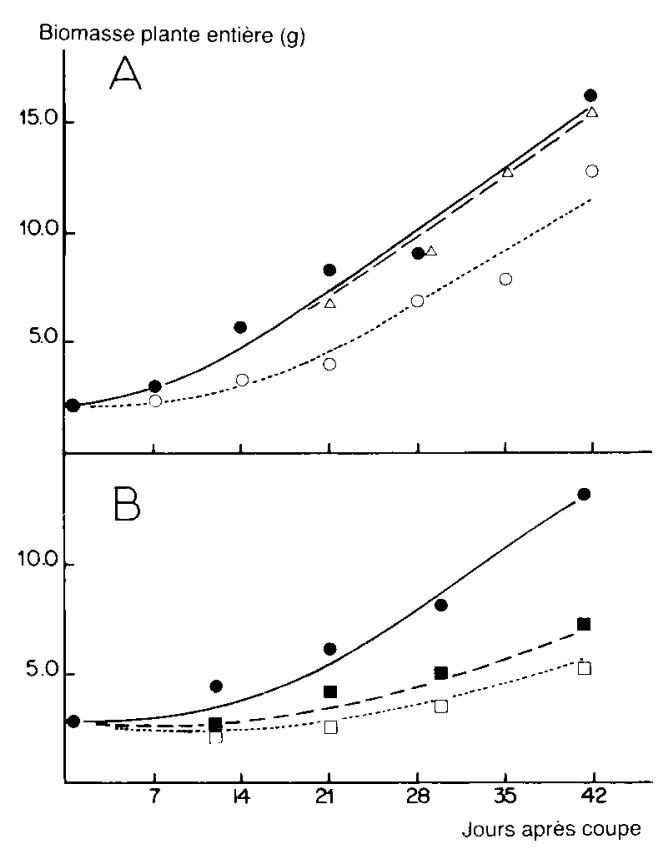

Figure 8

Comparaison des valeurs calculées (courbes continues) aux mesures de biomasse de la plante entière (symboles).

$A$ : essai I

$B$ : essai 2.

$\bullet:(E, N) ; \triangle:(E, N-n) ; O:(E, n)$;

$:(e, N) ; \square:(e, n)$

Comparison between the calculated values and the measurements of whole plant biomass.

$A$ : experiment $l$

$B$ : experiment 2 .

\section{DISCUSSION ET CONCLUSION}

L'ensemble des résultats permet de montrer les modalités par lesquelles la carence en azote modifie la vitesse de croissance d'un couvert en cours de repousse. 

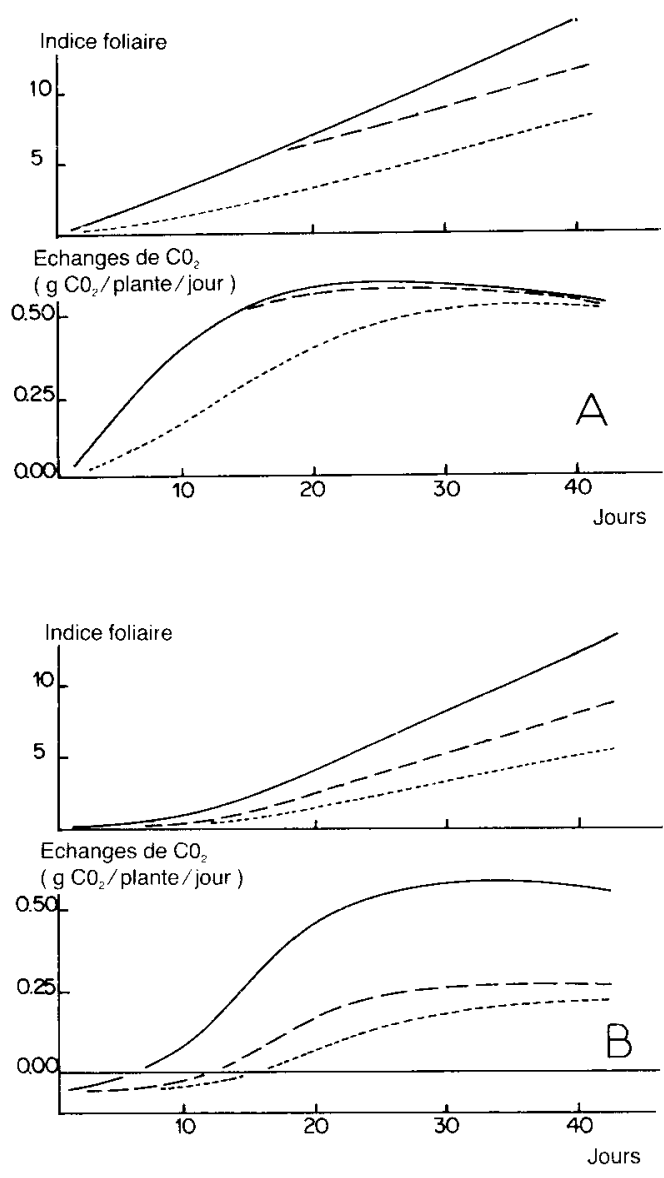

Figure 9

Evolution de l'indice foliaire et des échanges de $\mathrm{CO}_{2}$ calculés au cours de la croissance.

$A:$ Essai $1-:(E, N) ;--:(E, N-n) ; \cdots-\cdots \cdot(E, n)$.

$B:$ Essai $2-:(E, N) ;--:(e, N) \quad ;-\cdots-\cdots(e, n)$.

Leaf area index and calculated values of $\mathrm{CO}_{2}$ exchange during growth.

\section{Croissance de la plante entière}

- L'assimilation globale du couvert est affectée par la réduction de la vitesse de croissance de l'appareil aérien. La figure 9 montre que cet effet intervient en début de croissance mais qu'il est limité à la période où l'indice foliaire est inférieur à environ 6 pour l'éclairement $\mathrm{E}$ et à environ 4 pour l'éclairement $\mathrm{e}$, c'est-à-dire environ les $2 / 3$ de la durée de la repousse.

- Les capacités photosynthétiques du couvert ne sont pas affectées pendant la majeure partie de la repousse dans les conditions d'éclairement et de carence en azote où nous avons travaillé. Ce résultat, retrouvé par certains auteurs (WATSON, 1956) n'est pas toujours vérifié (ROBSON \& PARSONS, 1978). L'étude des capacités photosynthétiques de la feuille (GASTAL, 1984) permet d'expliquer cette controverse en montrant que l'effet de la carence en azote sur la fixation de $\mathrm{CO}_{2}$ d'un couvert dépend, d'une part, de l'intensité du déficit azoté des limbes mais, d'autre part, pour un indice foliaire donné, du niveau d'éclairement considéré.

- Les coûts respiratoires sont également modifiés. Le rapport respiration foliaire apparente/photosynthèse nette est nettement diminué en condition de carence en azote. Cependant, l'effet sur la respiration racinaire est plus difficile à interpréter puisque la diminution probable du coefficient d'entretien est compensée par l'augmentation de la biomasse et de la vitesse de croissance des racines.

\section{Croissance relative des appareils foliaire et racinaire}

Après la coupe apparaît un laps de temps pendant lequel la vitesse de croissance foliaire est ralentie. Au champ, ceci est également retrouvé par LEMAIRE $e t a l$. (comm. pers.) qui ont noté, à ce moment, une diminution de la vitesse d'émission des limbes sans toutefois de modification de leur vitesse d'élongation.

Dans nos essais, ce laps de temps diffère pour les traitements témoins de l'essai 1 et 2 et nous semble probablement lié au niveau des réserves carbonées au moment de la coupe.

Dans les conditions de faible intensité lumineuse, ce niveau de réserves dans les chaumes et les racines prend une importance particulière du fait d'un bilan carboné négatif pendant une longue période après la coupe : $15 \mathrm{j}$ pour le traitement (e, n) (fig. 9B). Sur une période de $20 \mathrm{j}$, la matière végétale photosynthétisée $(0,91 \mathrm{~g}$ MS) est approximativement égale à l'accroissement de biomasse aérienne (gaines + limbes : 0,85 g MS, fig. 2B). La diminution de biomasse des racines et des chaumes $(0,96 \mathrm{~g}$ MS) correspond à peu près au cumul de respiration de la plante entière $(0,84 \mathrm{~g}$ MS). L'importance de cette diminution (environ 35 p. 100 par rapport à la biomasse initiale) implique alors qu'en plus des molécules carbonées de réserve (fructosanes, glucides solubles) dont la teneur varie de 5 à 15 p. 100 selon les cas (BOOYSEN \& NELSON, 1975), d'autres composés carbonés aient été utilisés. DAVIDSON \& MILTHORPE (1966) ont déjà suggéré que protéines et hémi-celluloses puissent également jouer le rôle de substrat respiratoire.

La comparaison des mesures de croissance en matière sèche et des bilans de carbone pendant la repousse montre clairement que la carence en azote agit en tout premier lieu sur la croissance de la plante entière par une réduction de la vitesse de mise en place $d u$ système aérien. Elle indique également que ceci ne peut pas être interprété comme une simple modification de la répartition des assimilats entre système aérien et système racinaire en faveur de ce dernier : d'une part, la croissance racinaire du traitement carencé $(E, n)$ est inférieure à celle du traitement bien alimenté $(E, N)$ pendant toute la $1^{\text {re }}$ moitié de la repousse (fig. $2 \mathrm{C}$ ), et ce laps de temps est nettement plus long à éclairement plus faible (comparaison de $(e, N)$ et $(e, n)$, fig. 2D) ; d'autre part, si la carence en azote intervient non plus en début mais en milieu de repousse alors que l'assimilation de carbone est déjà importante $(\mathrm{E}, \mathrm{N}-\mathrm{n}$ ) (fig. $2 \mathrm{C}$ ), ce laps de temps est réduit à quelques jours. L'incidence de la déficience en azote sur la croissance différentielle système aérien/système racinaire est donc totalement dépendante de la fourniture en carbone. Ceci peut être interprété à partir des hypothèses suivantes :

a) le carbone disponible est utilisé en priorité pour la croissance aérienne,

$b$ ) la déficience en azote réduit la vitesse de croissance de l'appareil aérien, 
c) le carbone non utilisé par les parties aériennes est disponible pour la croissance du système racinaire.

Ces hypothèses sont vérifiées par les travaux de Ryle \& Powell (1976), Powell \& RYLe (1978) et ont été déjà proposées par GILLET et al. (1984).

On voit donc qu'il est possible de simuler l'assimilation de $\mathrm{CO}_{2}$ et la respiration d'un couvert de fétuque lorsqu'on connaît l'indice foliaire de ce couvert et l'éclairement reçu. Pour arriver à un modèle dynami- que de croissance en conditions contrôlées, il faut, en plus, simuler la répartition des assimilats entre les parties aériennes et souterraines de la plante. Les hypothèses ci-dessus peuvent servir de base à cette simulation, qui fera l'objet d'un travail ultérieur.

Reç le 5 avril 1985. Accepté le 27 septembre 1985.

\section{RÉFÉRENCES BIBLIOGRAPHIQUES}

Bassham J. A., Larsen P. O., Lawyer A. L., Cornwell K. L., 1981. Relationship between nitrogen metabolism and photosynthesis, 135163. In J. D. Bewley : "Nitrogen and carbon metabolism», Nijhoff-Junk, 248 p.

Blanc D., 1959. Contribution à l'étude de la nutrition azotée en culture sur sable. Ann. Physiol. Vég., 163-178.

Booysen P. de V., Nelson C. J., 1975. Leaf area and carbohydrate reserves in regrowth of tall fescue. Crop Sci., 15, 262-266.

Brouwer R., 1962. Distribution of dry matter in the plant. Neth. J. Agric. Sci., 10, 361-376.

Caloin M., El Khodre A., Atry M., 1980. Effect of nitrate concentration on the root : shoot ratio in Dactylis glomerata L. and on the kinetics of growth in the vegetative phase. Ann. Bot., 46, 165-173.

Chartier P., Bethenod O., 1977. La productivité primaire à l'échelle de la feuille, 77-112. In A. Moyse : "Les processus de la production végétale primaire », Gauthier-Villars, $265 \mathrm{p}$.

Clement C. R., Hopper M. J., Jones L. H. P., 1978. The uptake of nitrate by Lolium perenne from flowing nutrient solution. II. Effect of light, defoliation and relationship to $\mathrm{CO}_{2}$ flux. J. Exp. Bot., 29, 1173-1183.

Davidson J. L., Milthorpe F. L., 1966. The effect of defoliation on the carbon balance in Dactylis glomerata. Ann. Bot., 30, 128-185.

Davies A., 1965. Carbohydrate levels and regrowth in perennial ryegrass. J. Agric. Sci., 65, 213-221.

Deinum B., Dirven J. G. P., 1975. Climate, nitrogen and grass. 6. Comparison of yield and chemical composition of some temperate and tropical grass species grown at different temperatures. Neth. J. Agric. Sci., 23, 69-82.

Evans P. S., 1971. Root growth of Lolium perenne. II. Effects of defoliation and shading. N. Z. J. Agric. Res., 14, 552-562.

Evans J. R., 1983. Nitrogen and photosynthesis in the flag leaf of wheat. Plant Physiol., 72, 297-302.

Frossard J. S., 1980. L'éclairement du feuillage, facteur de régulation du rythme nycthéméral de la respiration des racines. Thèse $3^{\mathrm{e}}$ cycle, Clermont-Ferrand, $59 \mathrm{p}$.

Gastal F., 1984. Echanges de $\mathrm{CO}_{2}$ et croissance de la fétuque élevée en interaction avec la nutrition azotée. Thèse $3^{\mathrm{e}}$ Cycle, Orsay, $126 \mathrm{p}$.

Gastal F., Saugier B., 1986. Alimentation azotée et croissance de la fétuque élevée. II. Absorption de l'azote et distribution entre organes. Agronomie, 6 (4).

Gillet M., Lemaire G., Gosse G., 1984. Essai d'élaboration d'un schéma global de la croissance des graminées fourragères. Agronomie, 4 (1), 75-82.

Hunt R., 1975. Further observations on root-shoot equilibria in perennial ryegrass (Lolium perenne L.). Ann. Bot., 39, 745-755.
Jong T. M. de, 1982. Leaf nitrogen content and $\mathrm{CO}_{2}$ assimilation capacity in peach. J. Am. Soc. Hortic. Sci., 107, 955-959.

Lambers M., 1979. Efficiency of root respiration in relation to growth rate, morphology and soil composition. In « Energy Metabolism In Plants », Grassland species Research Group, Publ. $\mathrm{n}^{\circ} 13$, University of Groningen, Pays-Bas, $176 \mathrm{p}$.

Lemaire F., 1975. Action comparée de l'alimentation azotée sur la croissance du système racinaire et des parties aériennes des végétaux. Ann. Agron., 26 (1), 59-74.

McCree K. J., 1974. Equations for the rate of dark respiration of white clover and grain sorghum, as functions of dry weight, photosynthetic rate, and temperature. Crop Sci., 14, 509-514.

Monteith J. L., 1965. Light distribution and photosynthesis in field crops. Ann. Bot., 29, 17-37.

Oswalt D. L., Bertrand A. R., Teel M. R., 1959. Influence of nitrogen fertilization and clipping on grass roots. Soil Sci. Soc. Am. Proc., 23, 228-230.

Parsons A. J., Robson M. J., 1981. Seasonal changes in the physiology of S24 perennial ryegrass (Lolium perenne L.). 2. Potential leaf and canopy photosynthesis during the transition from vegetative to reproductive growth. Ann. Bot., 47, 249-258.

Penning de Vries F. W. T., 1975. The cost of maintenance in plant cells. Ann. Bot., 39, 71-92.

Powell C. E., Ryle G. J. A., 1978. Effect of nitrogen deficiency on photosynthesis and the partitioning of ${ }^{14} \mathrm{C}$-labelled leaf assimilate in unshaded and partially shaded plants of Lolium temulentum. Ann. appl. Biol., 90, 241-248.

Robson M. J., Parsons A. J., 1978. Nitrogen deficiency in smal closed communities of S24 ryegrass. I. Photosynthesis, respiration, dry matter production and partition. Ann. Bot., 42, 1185-1197.

Ruget F., 1981. Respiration de croissance et respiration d'entretien : méthodes de mesure, comparaison des résultats. Agronomie, 1, 601610 .

Ryle G. J. A., Powell C. E., 1976. Effect of rate of photosynthesis on the pattern of distribution in the gramineous plant. J. Exp. Bot., 27, 189-199.

Troughton A., 1956. Studies on the growth of young grass plants with special reference to the relationship between the shoot and root system. J. Br. Grassl. Soc., 11, 56-65.

Watson D. J., 1956. The physiological basis of variation in yield. Adv. Agron., 4, 101-144.

Woledge J., Leafe E. L., 1976. Single leaf and canopy photosynthesis in a ryegrass sward. Ann. Bot., 40, 773-783.

Yoshida S., Coronel V., 1976. Nitrogen nutrition, leaf resistance and leaf photosynthetic rate of the rice plant. Soil Sci. Plant Nutr., 22, 207-211. 